\title{
Relação entre índice de colheita do milho e eficiências de fósforo por meio de análise de trilha
}

\author{
Relationship between corn harvest index and phosphorus efficiencies through trail \\ Relación entre el índice de cosecha de maíz y las eficiencias de fósforo a través del análisis de
} senderos

\author{
Reginaldo Miranda de Oliveira \\ ORCID: https://orcid.org/0000-0002-1880-0730 \\ Universidade Federal de Viçosa, Brasil \\ E-mail: reginalo.miranda@ufv.br \\ Rubens Alves de Oliveira \\ ORCID: https://orcid.org/0000-0003-2557-592X \\ Universidade Federal de Viçosa, Brasil \\ E-mail: rubens@ufv.br \\ Julio Cesar Lima Neves \\ ORCID: https://orcid.org/0000-0001-8356-5100 \\ Universidade Federal de Viçosa, Brasil \\ E-mail: juliocneves@ufv.br \\ Ednaldo Miranda de Oliveira \\ ORCID: https://orcid.org/0000-0002-1182-7623 \\ Instituto Federal do Espírito Santo, Brasil \\ E-mail: ednaldo.oliveira@ifes.edu.br \\ Margareth Evangelista Botelho \\ ORCID: https://orcid.org/0000-0001-8746-2272 \\ Universidade Federal de Viçosa, Brasil \\ E-mail: margareth.botelho@ufv.br \\ Job Teixeira de Oliveira \\ ORCID: https://orcid.org/0000-0001-9046-0382 \\ Universidade Federal do Mato Grosso do Sul, Brasil \\ E-mail: job.oliveira@hotmail.com
}

\begin{abstract}
Resumo
A produtividade das diversas culturas, incluindo o milho, é influenciada pela habilidade das plantas em absorver e utilizar nutrientes. O objetivo foi verificar, por meio da análise de trilha, as relações diretas e indiretas entre o índice de colheita e as eficiências de uso de fósforo no milho. As variáveis do estudo foram: índice de colheita do milho (HI), índice de colheita para fósforo (FO), eficiência de uso compartimentalizado na folha (CF), eficiência de uso compartimentalizado no caule (CC), eficiência de uso compartimentalizado na palha (CP), eficiência de uso compartimentalizado no grão (CG), eficiência de uso integralizado na folha (IF), eficiência de uso integralizado no caule (IC), eficiência de uso integralizado na palha (IP). Por meio da correlação de Pierson, observamos correlação alta e positiva entre CP e IP, CF e IF e IC e FO. Houve correlação forte e negativa entre: IC e IP, IC e CP e FO e CG. O Concluiu-se que as variáveis que obtiveram os maiores efeitos diretos e positivos sobre o índice de colheita do milho foram: índice de colheita para fosforo e eficiência de uso compartimentalizado no grão. Efeitos diretos e negativos sobre o índice de colheita do milho foram: eficiência de uso compartimentalizado na palha e eficiência de uso compartimentalizado na folha. De forma indireta, a análise de trilha mostrou que eficiência de uso compartimentalizado no grão e eficiência de uso integralizado na folha são fortemente correlacionados positivamente, e no sentido oposto, eficiência de uso compartimentalizado no grão e índice de colheita para fósforo são correlacionados negativamente.

Palavras-chave: Estatística multivariada; Nutrientes; Adubação; Fertirrigação; Zea Mays.
\end{abstract}

\begin{abstract}
The productivity of different crops, including corn, is influenced by the ability of plants to absorb and use nutrients. The objective was to verify, through trail analysis, the direct and indirect relationships between the harvest index and the efficiency of phosphorus use in corn. The study variables were: corn harvest index (HI), phosphorus harvest index (FO), compartmentalized use efficiency on the leaf (CF), compartmentalized use efficiency on the stem (CC), compartmentalized use efficiency on the straw (CP), efficiency of use compartmentalized in the grain (CG), efficiency of use integrated in the leaf (IF), efficiency of use integrated in the stem (IC), efficiency of use integrated in the straw (IP). Through Pierson's correlation, we observed a high and positive correlation between CP and IP, CF and IF and IC and FO. There was a strong and negative correlation between: IC and IP, IC and CP and FO and CG. O It was concluded
\end{abstract}


that the variables that obtained the greatest direct and positive effects on the corn harvest index were: harvest index for phosphorus and efficiency of compartmentalized use in the grain. Direct and negative effects on the corn harvest index were: efficiency of compartmentalized use in the straw and efficiency of compartmentalized use in the leaf. Indirectly, the trail analysis showed that efficiency of compartmentalized use in the grain and efficiency of integral use in the leaf are strongly positively correlated, and in the opposite direction, efficiency of compartmentalized use in the grain and harvest index for phosphorus are negatively correlated.

Keywords: Multivariate statistics; Nutrients; Fertilization; Fertigation; Zea Mays.

\begin{abstract}
Resumen
La productividad de diferentes cultivos, incluido el maíz, está influenciada por la capacidad de las plantas para absorber y utilizar nutrientes. El objetivo fue verificar, a través del análisis de rastros, las relaciones directas e indirectas entre el índice de cosecha y la eficiencia del uso de fósforo en maíz. Las variables de estudio fueron: índice de cosecha de maíz (HI), índice de cosecha de fósforo (FO), eficiencia de uso compartimentado en la hoja (CF), eficiencia de uso compartimentado en el tallo (CC), eficiencia de uso compartimentado en la paja (CP), eficiencia de uso compartimentado en el grano (CG), eficiencia de uso integrada en la hoja (IF), eficiencia de uso integrada en el tallo (IC), eficiencia de uso integrada en la paja (IP). A través de la correlación de Pierson, observamos una correlación alta y positiva entre CP e IP, CF e IF e IC y FO. Hubo una correlación fuerte y negativa entre: IC e IP, IC y CP y FO y CG. O Se concluyó que las variables que obtuvieron mayores efectos directos y positivos sobre el índice de cosecha de maíz fueron: índice de cosecha de fósforo y eficiencia de uso compartimentado en el grano. Los efectos directos y negativos sobre el índice de cosecha de maíz fueron: eficiencia del uso compartimentado en la paja y eficiencia del uso compartimentado en la hoja. Indirectamente, el análisis de rastros mostró que la eficiencia del uso compartimentado en el grano y la eficiencia del uso integral en la hoja están fuertemente correlacionadas positivamente, y en la dirección opuesta, la eficiencia del uso compartimentado en el grano y el índice de cosecha de fósforo están correlacionados negativamente.
\end{abstract}

Palabras clave: Estadística multivariante; Nutrientes; Fertilización; Fertirrigación; Zea Mays.

\title{
1. Introdução
}

A cultura do milho é bastante adaptada às condições tropicais brasileiras, cuja produção em 2020, considerando as três safras, atingiu recorde na série histórica com total de 102,1 milhões de toneladas, aumento de 2,1\% em relação ao exercício anterior (CONAB, 2020). Essa elevada produção deve-se, em parte, ao uso de genótipos adaptados às condições de solo e clima do país, mas também aos tratos culturais realizados, sendo merecidos maiores destaques à adubação e à irrigação.

A influência da planta na eficiência da adubação de fosforo, materiais genéticos selecionados que tenham boa adaptação a condições de fertilidade baixa do solo pode aumentar o aproveitamento dos fertilizantes aplicados sensivelmente (Luca et al., 2002). É definida como a relação entre produção e a concentração do nutriente no tecido, a eficiência de uso de um nutriente enquanto que para Graham (1984), eficiência é a aptidão de um genótipo ou espécie em fornecer altas produtividades em um solo deficiente no nutriente em estudo.

O coeficiente de correlação de Pearson é uma ferramenta bastante utilizada para expressar, por meio de um valor puro, a associação entre duas variáveis quantitativas. Entretanto, essa medida é insuficiente para explicar uma relação de causa e efeito (Blind et al., 2018), uma vez que outras podem estar interferindo nessa relação (Oliveira et al., 2018; Sari et al., 2018), conduzindo o pesquisador a conclusões errôneas pela obtenção de valores superestimados.

Uma forma de remover o efeito de outras variáveis sobre a relação de um par de variáveis pode ser por meio da análise de trilha. A análise trilha tem a capacidade de particionar o coeficiente de correlação e quantificar as relações diretas e indiretas de todas as variáveis envolvidas na resposta de uma (GONÇALVES et al., 2017).

Com base no exposto, o estudo foi desenvolvido com o objetivo de verificar por meio da análise de trilha, as relações diretas e indiretas entre o índice de colheita e as eficiências de fósforo no milho.

\section{Material e Métodos}

Utilizando uma área total de 1,08 ha sob um pivô central localizado no município de Coimbra - MG (2049’51', $\mathrm{S}$ e $42^{\circ} 48^{\prime} 10^{\prime \prime}$ ), foi realizado, entre os meses de janeiro/2018 e maio/2018 um ciclo da cultura de milho. A altitude média da área 
de cultivo é de $716 \mathrm{~m}$ em relação ao nível do mar. A temperatura média e a precipitação média anual são de $19^{\circ} \mathrm{C}$ e $1.300 \mathrm{~mm}$, respectivamente. Com período chuvoso e quente na primavera-verão e período seco e frio no outono-inverno. Segundo análise química do solo (Tabela 1), observamos os teores de nutrientes do dolo da área de cultivo, que foi classificado como Argissolo Vermelho-Amarelo distrófico (EMBRAPA, 2018).

Tabela 1 - Características químicas do solo da área experimental.

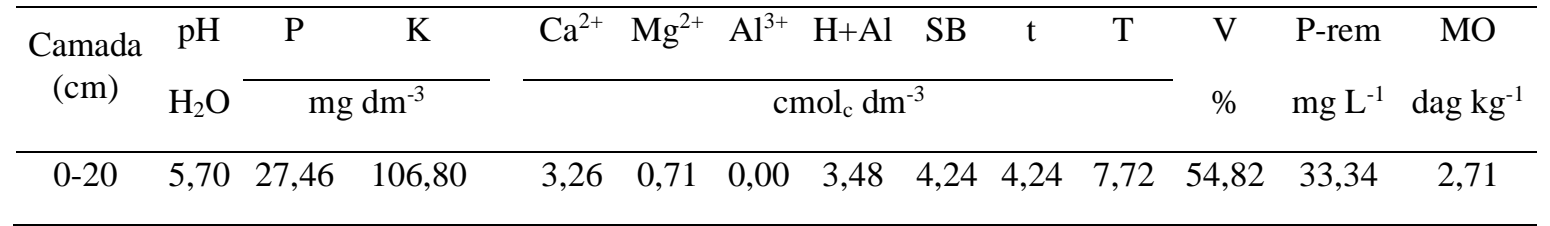

$\mathrm{P}, \mathrm{Na}, \mathrm{K}$ - Extrator Mehlich - 1. H+Al - acetato de cálcio 0,5 mol L-1. Ca, Mg, Al - KCl 1 mol L-1. Mat. Org.(MO) - C.org. x 1,724. SB: Soma de bases. t: CTC efetiva. T: CTC a pH 7,0. V: Saturação de bases. Fonte: Autores.

O híbrido simples de milho DBK390 PROIII, super precoce, foi cultivado em linhas espaçadas de 0,50 m. Foi realizado o plantio de aproximadamente 100.000 plantas por hectares e posterior desbaste para garantir o estande de 60.000 e 80.000 plantas por hectare, nas P60 e P80, respectivamente. No momento do plantio foram aplicados fósforo nas doses 0, 50, 100 e 200 $\mathrm{kg} \mathrm{ha}^{-1}$ (D0, D50, D100 e D200, respectivamente) de $\mathrm{P}_{2} \mathrm{O}_{5}$, utilizando o adubo superfosfato triplo. Em cobertura, quando as plantas de milho se encontravam nos estádios fenológicos V3 e V6, apresentando três e seis folhas totalmente formadas, respectivamente, foram aplicado nitrogênio $\left(170 \mathrm{~kg} \mathrm{ha}^{-1}\right)$ e potássio $\left(120 \mathrm{~kg} \mathrm{ha}^{-1}\right)$, dividido igualmente nas duas adubações. Os demais tratos culturais ocorreram de acordo com o recomendado para a cultura do milho na região.

A demanda hídrica diária foi determinada com base na evapotranspiração da cultura, obtida a partir dos parâmetros climáticos coletados por uma estação meteorológica automática da marca DAVIS, modelo Vantage Pro II instalada próxima à área de cultivo. A área sob o pivô foi dividida em 7 talhões nos quais variou-se a frequência de irrigação, sendo utilizados 1, 2, 3, 4, 5, 6 e 7 dias de turno de rega (TR1, TR2, TR3, TR4, TR5, TR6 e TR7, respectivamente). O total de água aplicado em cada turno de rega correspondeu à soma da demanda hídrica dos dias sem irrigação.

O desenho experimental utilizado foi um esquema de parcelas sub-subdivididas, no qual os turnos de rega ocupavam as parcelas, as densidades de plantio (populações) estavam nas subparcelas e as doses de adubação nas sub-subparcelas. Cada tratamento foi conferido à uma unidade experimental, cuja área útil correspondia a 7,50 $\mathrm{m}^{2}$, composta por 5 linhas de plantio com 5 metros de comprimento, sendo que as linhas das extremidades foram consideradas como bordaduras.

Após o encerramento do ciclo da cultura, quando os grãos estavam maduros e secos, toda a parte aérea de uma planta representante de cada unidade experimental foi colhida para determinação dos teores de matéria seca, de fósforo e, posterior estimação das eficiências de utilização do fósforo. Além disso, para determinação da produtividade foram colhidas todas as espigas da área útil de cada unidade experimental. A produtividade foi estimada em $\mathrm{kg} \mathrm{ha}^{-1}$, corrigida para $13 \%$ de umidade e utilizada para determinar as variáveis deste estudo, conforme segue:

- Índice de colheita do milho (HI). Trata-se da divisão do conteúdo de produtividade do milho grão, na colheita, pelo conteúdo de matéria seca da parte aérea da planta de milho na colheita. Em $\mathrm{kg} \mathrm{kg}^{-1}$.

- $\quad$ Índice de colheita para fósforo (FO). É a divisão do conteúdo de fósforo no milho grão na colheita pelo conteúdo de fosforo na parte aérea da planta de milho na colheita. Em $\mathrm{kg} \mathrm{kg}^{-1}$.

- $\quad$ Eficiência de uso compartimentalizado na folha (CF). Refere-se à divisão do conteúdo de matéria seca da folha pela quantidade de fósforo contido na folha da planta de milho. Em $\mathrm{kg} \mathrm{kg}^{-1}$.

- $\quad$ Eficiência de uso compartimentalizado no caule (CC). Divisão do conteúdo de matéria seca no caule pela quantidade de fósforo contido no caule da planta de milho. Em $\mathrm{kg} \mathrm{kg}^{-1}$. 
- $\quad$ Eficiência de uso compartimentalizado na palha (CP). Refere-se à divisão do conteúdo de matéria seca da palha da espiga pela quantidade de fósforo contido na palha da espiga de milho. Em $\mathrm{kg} \mathrm{kg}^{-1}$.

- $\quad$ Eficiência de uso compartimentalizado no grão (CG). Refere-se à divisão do conteúdo de matéria seca do grão da espiga pela quantidade de fósforo contido no grão da espiga de milho. $\mathrm{Em} \mathrm{kg} \mathrm{kg}^{-1}$.

- $\quad$ Eficiência de uso integralizado na folha (IF). É a divisão da massa seca da folha pela quantidade de fósforo contido na planta total de milho. Em kg kg-1.

- $\quad$ Eficiência de uso integralizado no caule (IC). Refere-se a divisão da massa seca do caule pela quantidade de fósforo contido na planta total de milho. $\mathrm{Em} \mathrm{kg} \mathrm{kg}^{-1}$.

- $\quad$ Eficiência de uso integralizado na palha (IP). Refere-se a divisão da massa seca da palha da espiga pela quantidade de fósforo contido na planta total de milho. Em $\mathrm{kg} \mathrm{kg}^{-1}$.

$\mathrm{O}$ grau de multicolinearidade da matriz $\mathrm{X}^{\prime} \mathrm{X}$ foi estabelecido com base no número de condição (NC) que é obtido pela razão entre o maior e o menor autovalores da matriz de correlações (MONTGOMERY et al., 2012). Se NC<100, a multicolinearidade é considerada fraca e não representa problema para a análise; Se $100 \leq \mathrm{NC} \leq 1.000$ implica multicolinearidade moderada a forte e; se NC>1.000 a multicolinearidade é considerada severa. Para resolver o problema da multicolinearidade, e proceder a análise de trilha sem que houvesse a necessidade de remover qualquer componente envolvido no estudo foi adicionada uma constante $(k=0,05)$ à diagonal da matriz de correlações.

Tomando o índice de colheita como dependente principal, procedeu-se a análise de trilha, para particionar as correlações das demais variáveis com o índice de colheita em relações diretas e indiretas. Para as análises de correlação e diagnóstico de multicolinearidade foi utilizado o Software estatístico RBio (BHERING, 2017). Para a análise de trilha foi utilizado o Software estatístico GENES (CRUZ, 2013). A metodologia da escrita do presente trabalho seguiu as diretrizes de Pereira et al. (2018).

\section{Resultados e Discussão}

Na Figura 1 está expressa a rede de correlações de Pearson entre as variáveis do estudo. As estimativas obtidas a partir da matriz de correlações, oscilaram entre $-0,4449 *$ a $0,7261 * *$. 
Figura 1 - Rede de correlações das variáveis: índice de colheita do milho (HI), índice de colheita para fosforo (FO), eficiência de uso compartimentalizado na folha (CF), eficiência de uso compartimentalizado no caule (CC), eficiência de uso compartimentalizado na palha (CP), eficiência de uso compartimentalizado no grão (CG), eficiência de uso integralizado na folha (IF), eficiência de uso integralizado no caule (IC), eficiência de uso integralizado na palha (IP).

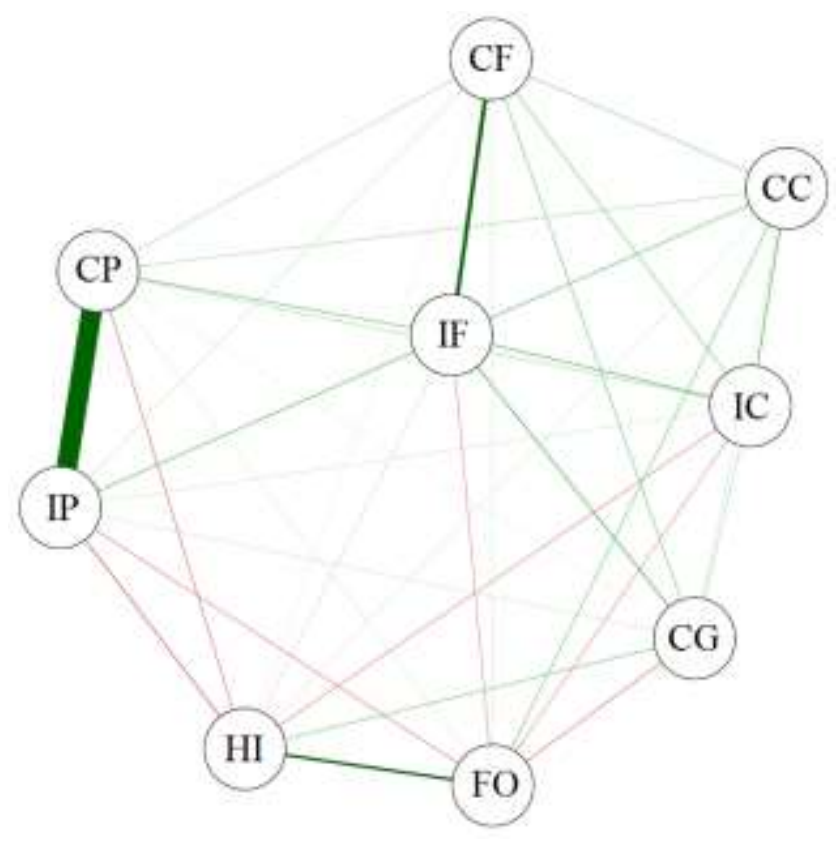

Fonte: Autores.

Houve correlação alta e positiva entre CP e IP, CF e IF e IC e FO. Estes resultados mostram que há estreita relação entre eficiência de uso compartimentalizado e uso integralizado, como podemos observar nestas verificações na palha e na folha. Houve correlação forte e negativa entre: IC e IP, IC e CP e FO e CG. Estes resultados sugerem que aumento de concentração de fósforo na palha, refletem em menor concentração de fósforo no caule, e vice versa. Vitorino et al. (2020), verificaram aumentos de teores de fósforo no caule com o aumento de doses de fósforo aplicadas no solo.

De acordo com o critério NC apresentado por Montgomery et al. (2012), a matriz de estimativa das correlações de Pearson obtidas aqui apresentaram multicolinearidade moderada a forte com um número de condição $=170$. Portanto, foi realizada análise de trilha em crista para corrigir a multicolineariedade.

Neste trabalho, a apresentação dos resultados foi feita por meio de um esquema (Figura 2), onde é possível verificar as variáveis que obtiveram os maiores efeitos diretos e positivos sobre o índice de colheita do milho (HI) que foram: índice de colheita para fosforo (FO) e eficiência de uso compartimentalizado no grão (CG), com valores de correlação de 0,9047 e 0,6171, respectivamente. Efeitos diretos e negativos sobre o índice de colheita do milho (HI) foram: eficiência de uso compartimentalizado na palha (CP) e eficiência de uso compartimentalizado na folha (CF), com valores de correlação de -0,3704 e - 0,2819 , respectivamente. Estes resultados mostram que aumento do teor de fósforo na palha e na folha, diminuem o índice de colheita do milho. Vitorino et al. (2020), relataram que quantidades acumuladas de P nas folhas aumentaram de forma linear no tratamento com fosfito via foliar. 
Figura 2 - Análise de trilha entre índice de colheita do milho (HI) e demais variáveis do estudo: índice de colheita para fosforo (FO), eficiência de uso compartimentalizado na folha (CF), eficiência de uso compartimentalizado no caule (CC), eficiência de uso compartimentalizado na palha (CP), eficiência de uso compartimentalizado no grão (CG), eficiência de uso integralizado na folha (IF), eficiência de uso integralizado no caule (IC), eficiência de uso integralizado na palha (IP).

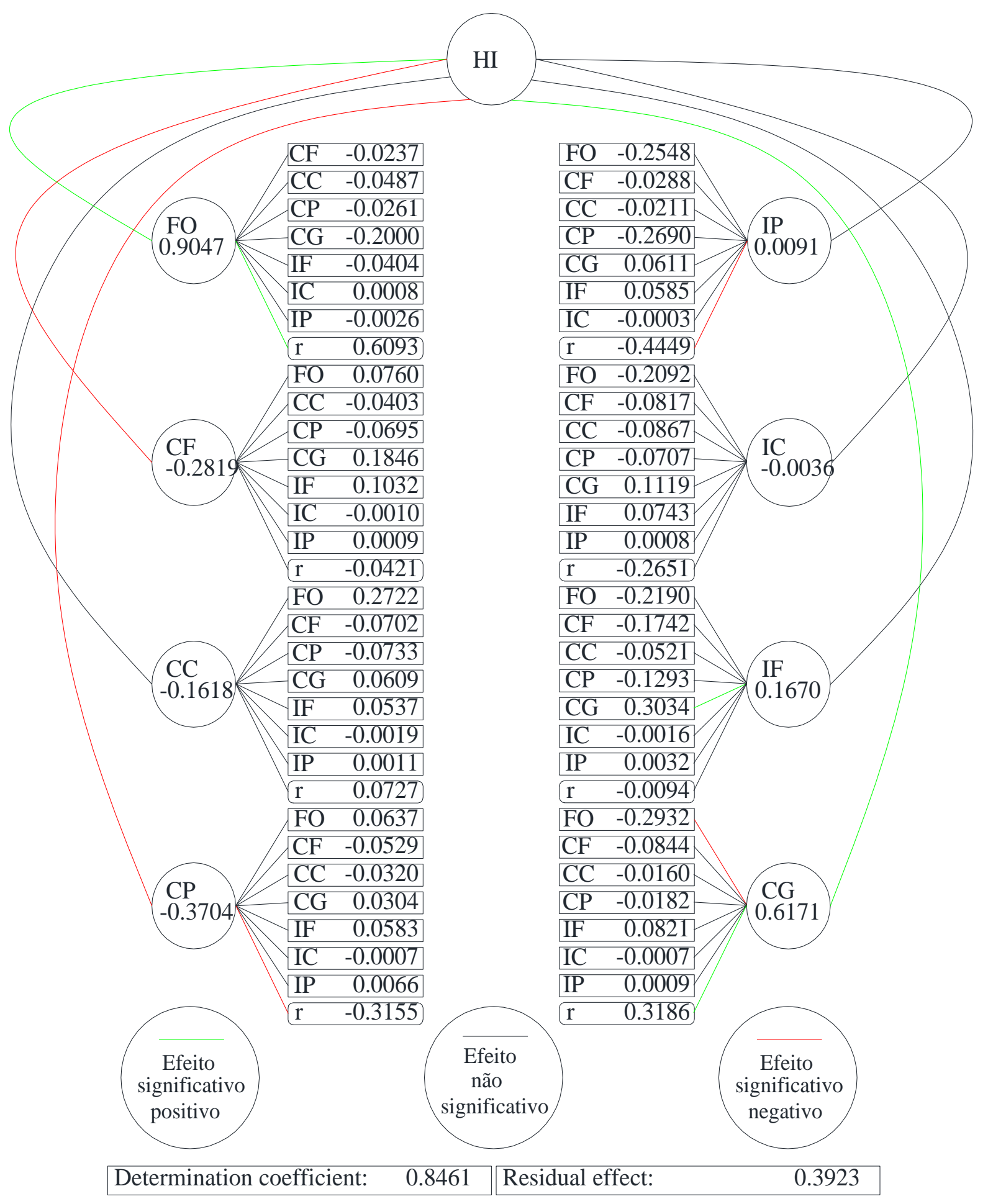

Fonte: Autores.

Este resultado também sugere de forma indireta, que eficiência de uso compartimentalizado no grão (CG) e eficiência de uso integralizado na folha (IF) são fortemente correlacionados de forma positiva. No sentido oposto, eficiência de uso compartimentalizado no grão (CG) e índice de colheita para fosforo (FO) são correlacionados negativamente. 
Neste estudo foi obtido um alto coeficiente de determinação $(0,8461)$ que, por sua vez, pode ser implementado para melhoria e valorização desta importante cultura de grãos, através de estudos mais aprimorados no manejo do milho. Pinheiro et al. (2021), encontraram coeficiente de determinação de 0,7544 em estudo com atributos físicos do milho via análise de trilha.

A diferença entre espécies na absorção de fósforo foi citada em vários estudos e há um consenso que na absorção estão a dinâmica de crescimento da planta, características do sistema radicular, afinidade íon-carregador e transformações químicas que ocorrem na interface solo-raíz (LUCA et al., 2002). Estes autores também relatam que o local de origem das plantas é um fator importante na capacidade de absorção e utilização de fósforo. Algumas espécies de plantas que crescem em regiões de solos pobres em fósforo, parecem ser muito eficientes na sua utilização e pouco eficientes na absorção de fósforo.

\section{Conclusão}

Por meio da análise de trilha, foi possível verificar as variáveis que obtiveram os maiores efeitos diretos e positivos sobre o índice de colheita do milho foram: índice de colheita para fosforo e eficiência de uso compartimentalizado no grão. Efeitos diretos e negativos sobre o índice de colheita do milho foram: eficiência de uso compartimentalizado na palha e eficiência de uso compartimentalizado na folha.

De forma indireta, a análise de trilha mostrou que eficiência de uso compartimentalizado no grão e eficiência de uso integralizado na folha são fortemente correlacionados positivamente, e no sentido oposto, eficiência de uso compartimentalizado no grão e índice de colheita para fosforo são correlacionados negativamente.

Sugere-se mais estudos nesta área, pois a estatística multivariada pode agregar muitos conhecimentos na cultura do milho, e por isso, resultados nesta linha, com outros atributos podem ajudar no melhor entendimento da produção da cultura.

\section{Agradecimentos}

Este estudo foi financiado em parte pelo Coordenação de Aperfeiçoamento de Pessoal de Nível Superior - Brasil (CAPES) - Finance Code 001.

Este trabalho foi realizado com o apoio de CNPq, Conselho Nacional de Desenvolvimento Científico e Tecnológico Brazil. 141231/2019-0.

À Fundação Arthur Bernardes (FUNARBE), pelo apoio financeiro para publicação deste trabalho. À Universidade Federal do Mato Grosso do Sul (UFMS, Chapadão do Sul, MS) e à Universidade Federal de Viçosa (UFV, Viçosa, MG).

\section{Referências}

Bhering, L. L. (2017). RBio: A Tool For Biometric and Statistical Analysis Using The R Platform. Crop Breeding And Applied Biotechnology, 17, 187 - 190. https://doi.org/10.1590/1984-70332017v17n2s29.

Blind, A. D., Valente, M. S. F., Lopes, M. T.G., \& Resende, M. D. V. (2018). Estimativa de parâmetros genéticos, análise de trilha e seleção em bucha vegetal para caracteres agronômicos. Revista Brasileira de Ciências Agrárias, 13(2). 10.5039/agraria.v13i2a5522.

CONAB - Companhia Nacional de Abastecimento. (2020). Acompanhamento da safra brasileira de grãos, v. 7 - Safra $2019 / 20$ - Décimo primeiro levantamento, Brasília, p. 1 - 62, agosto 2020. https://www.conab.gov.br/info-agro/safras/graos/boletim-da-safra-de-graos.

Cruz, C.D. Genes: (2013). A Software Package for Analysis in Experimental Statistics and Quantitative Genetics. Acta Scientiarum: Agronomy, 35,271 - 276. https://doi.org/10.4025/actasciagron.v35i3.21251.

EMBRAPA - Empresa Brasileira de Pesquisa Agropecuária. (2017). Manual de métodos de análise de solo. $3^{\mathrm{a}}$ edição revista e ampliada. Brasília, DF: Embrapa. EMBRAPA - Empresa Brasileira de Pesquisa Agropecuária. (2018). Sistema brasileiro de classificação de solos. Brasília, DF: Embrapa. (5a ed.), revista e ampliada.

Gonçalves, D. L., Barelli, M. A. A., Oliveira, T. C., Santos, P. R. J., Silva, C. R., Poletine, J. P., \& Neves, L. G. (2017). Genetic correlation and path analysis of common bean collected from Caceres Mato Grosso State, Brazil. Ciência Rural, 47(8). https://doi.org/10.1590/0103-8478cr20160815.

Graham, R. D. (1984). Breeding for nutritional characteristics in cereals. In: Tinker, P. B., \& Lauchli, A. (Ed.) Advances in plant nutrition. New York: Praeger, 57-102. 
Research, Society and Development, v. 10, n. 2, e13510212358, 2021

(CC BY 4.0) | ISSN 2525-3409 | DOI: http://dx.doi.org/10.33448/rsd-v10i2.12358

Luca, E. F. D., Boaretto, A. E., Muraoka, T., \& Chitolina, J. C. (2002). Eficiência de absorção e utilização de fósforo (32P) por mudas de eucalipto e arroz. Scientia Agricola, 59 (3), 543-547. https://doi.org/10.1590/S0103-90162002000300020.

Montgomery, D. C., Peck, E. A., \& Vining, G.G. (2012). Introduction to Linear Regression Analysis. 5. ed. Wiley: New York, 672 p.

Oliveira, J. T., Ribeiro, I. S., Roque, C. G., Montanari, R., Gava, R., \& Teodoro, P. E. (2018). Contribution of morphological traits for grain yield in common bean. Bioscience Journal, 34 (2). https://doi.org/10.14393/BJ-v34n2a2018-39701.

Pereira, A. S., Shitsuka, D. M., Parreira, F. J., Shitsuka, R. (2018). Metodologia da pesquisa científica. UAB/NTE/UFSM.https://repositorio.ufsm.br/bitstrea $\mathrm{m} /$ handle/1/15824/Lic_Computacao_Metodologia-Pesquisa-Cientifica.pdf?sequence=1.

Pinheiro, L. S., Silva, R. C., Vieira, R. C., Aguiar, R. O., Nascimento, M. R., Vieira, M. M., \& Silva, P. A. (2021). Análise de trilha dos atributos físicos de milho (Zea mays L.) em sistema de cultivo convencional. Research, Society and Development, 10 (1). https://doi.org/10.33448/rsd-v10i1.10832.

Sari, B. G., Lúcio, A. D., Olivoto, T., Krysczun, D. K., Tischler, A. L., \& Drebes, L. (2018). Interference of sample size on multicollinearity diagnosis in path analysis. Pesquisa Agropecuária Brasileira, v. 53, n. 6, p. 769-773. https://doi.org/10.1590/s0100-204x2018000600014.

Vitorino, P. J. P., Santos, E. N., Rocha, J. L. A., Marcelino, R. M. O. S., \& Santos, L. C. (2020). Crescimento e acúmulo de fósforo em milho sob doses de fosfato radicular e fosfito via foliar. Research, Society and Development, 9 (5). https://doi.org/10.33448/rsd-v9i5.3120. 